\title{
Standard Operating Procedures - Schnittstellen zur Biometrie aus der Sicht des Sponsors
}

\author{
Werner Pölz, Wolfgang Schimetta und Gabriele Pölz \\ Institut für Systemwissenschaften, Johannes Kepler Universität Linz
}

Zusammenfassung: Verfahrensanleitungen (SOPs) dienen zur Standardisierung von Vorgangsweisen. Indem sie ständig aktualisiert werden, tragen sie wesentlich zur Qualitätsoptimierung bei. In klinischen Studien sollten SOPs von allen beteiligten Parteien (Prüfarzt, Sponsor, Biometriker) verwendet werden. Der Prüfarzt (Prüfer) gewährleistet damit u.a. die Wahrung der Sicherheit und der Rechte der Studienteilnehmer (Versuchspersonen), die qualitativ einwandfreie und möglichst vollständige Datenerhebung und -dokumentation sowie die Einhaltung der Rechtsnormen. Der Sponsor verwendet SOPs für die Normierung von Planungs- und (Qualitäts-)Überwachungsaktivitäten, für Melde-, Versicherungs- und Dokumentationspflichten sowie ebenfalls für die Einhaltung der Rechtsnormen. Ein bewährtes Modell stellt die Positionierung des Biometrikers als dritte autonome Partei neben dem Prüfer und dem Sponsor dar. Dementsprechend sind die Verantwortlichkeitsbereiche klar abzugrenzen und Schnittstellen in Form von Anforderungen an die jeweilige andere Partei (bezüglich Qualifikation, Ressourcen, internes Qualitätsmanagement etc.) zu definieren.

\begin{abstract}
Standard operating procedures (SOPs) are detailed, written instructions for the management of clinical trials. They provide a framework enabling the efficient implementation and performance of all the functions and activities for each clinical study. As an essential instrument for quality assurance, they should be used by all major parties involved in the conduct and evaluation of clinical trials, i.e. sponsors, investigators and biometricians. The article presents a three-party-model for the co-operation in clinical trials and outlines the aspects to be considered when drawing up an SOP defining the requirements to be fulfilled by the biometrician.
\end{abstract}

Schlüsselwörter: Biometrie, SOPs, Standard Operating Procedures, Good Clinical Practice, GCP, Klinische Studien.

\section{Einleitung}

Standard Operating Procedures (SOPs) sind eingehende, schriftlich festgelegte Anweisungen, mit denen die im Rahmen einer klinischen Studie durchzuführenden Verfahren operationalisiert und standardisiert werden. Sie stellen somit ein internes - d.h. beim jeweiligen Experten etabliertes - Regelwerk dar, das für die Einhaltung des Qualitätsstandards der Guten Klinischen Praxis (Good Clinical Practice - kurz GCP) unerläßlich ist.

Das Konzept der Guten Klinischen Praxis wurde in einer EG-Leitlinie mit dem Titel „Gute Klinische Praxis für die klinische Prüfung von Arzneimitteln in der Europäischen Gemeinschaft" aus dem Jahr 1991 ausführlich beschrieben. Es handelt sich bei der Guten 
Klinischen Praxis um einen Standard, nach welchem klinische Prüfungen geplant, durchgeführt und berichtet werden, so daß sichergestellt ist, daß die Daten glaubwürdig sind und daß die Rechte und die Unversehrtheit der Personen sowie die Vertraulichkeit der Daten geschützt sind.

Der Standard der Guten Klinischen Praxis ist jedoch keinesfalls eine europäische Domäne. Die Internationale Harmonisierungskonferenz - ICH -, deren Mitglieder die Europäische Union, USA und Japan sind, hat 1996 ebenfalls eine „Guideline for Good Clinical Practice“, die über eine Zusammenführung der Qualitätsnormen aller drei Regionen auch eine Weiterentwicklung der EG-Leitlinie darstellt, und in allen Vertragsstaaten im Sinne einer Empfehlung zu beachten ist, verabschiedet. Die Gute Klinische Praxis ist also ein internationaler Qualitätsstandard.

SOPs dienen der Einhaltung dieses Standards und sind somit essentiell ein Instrument der Qualitätssicherung. Die Verbindlichkeit der Verwendung von SOPs ergibt sich in Österreich aus dem Arzneimittelgesetz (AMG). Durch die AMG-Novelle 1994 wurde nämlich das EG-GCP-Konzept, das einen klaren Auftrag im Hinblick auf Qualitätssicherungsmaßnahmen enthält, in die österreichische Rechtsordnung implementiert. $\S 31$ AMG normiert dementsprechend: ,Der Sponsor hat detaillierte Verfahrensvorschriften (SOPs) zu erarbeiten und für deren Einhaltung Sorge zu tragen.Normadressat in Bezug auf die Qualitätssicherung ist laut AMG generell der Sponsor.

Die Qualitätssicherungsmaßnahmen umfassen jedoch nicht nur interne Verfahrensvorschriften, also SOPs, sondern u.a. auch die Bestimmungen zur Gewährleistung der Qualifikation der an der klinischen Studie mitwirkenden Fachkräfte. Der Sponsor hat gemäß $\S 37$ Abs 5 AMG „einen entsprechend qualifizierten Biometriker oder Statistiker“ mit der Planung der statistischen Analyse und ihrer Ausführung zu betrauen (die erwähnte ICH-GCP-Guideline fordert in diesem Zusammenhang sogar einen ,entsprechend qualifizierten und erfahrenen Statistiker“). Wenn $§ 47$ Abs 1 AMG darlegt: „Alle Lenkungsmaßnahmen“ (im Bereich der Qualitätskontrolle und Qualitätssicherung), „müssen auf die Verhütung unzulänglicher Qualität abzielen.“, so wird der Sponsor bei der Beurteilung der „entsprechenden Qualifikation“ des Biometrikers sehr wohl darauf zu achten haben, inwieweit dieser selbst Instrumente zur Qualitätssicherung etabliert und installiert hat.

\section{Kooperationsmodelle für klinische Prüfungen}

Prinzipiell sieht das Konzept der GCP zwei hauptverantwortliche Personen oder Parteien für die Abwicklung von klinischen Prüfungen vor: den Prüfer (bzw. Prüfarzt) und den Sponsor. Laut AMG ist der Prüfer der Arzt, der die klinische Prüfung praktisch durchführt und die damit im Zusammenhang stehende Verantwortung für die Versuchspersonen trägt, während der Sponsor als physische oder juristische Person die Verantwortung für Planung, Betreuung und Finanzierung selbigen Projekts übernimmt.

Die im AMG und in der ICH-GCP-Guideline ausführlich dargestellte Aufteilung der Verantwortlichkeiten zwischen Prüfer und Sponsor wurde primär für eine Konstellation entwickelt, in der ein potenter und fachkundiger Auftraggeber (wie insbesondere ein Pharmakonzern) mit einem (naturgemäß ebenfalls hochqualifizierten) Prüfer zusammenarbeitet. 
Darüber hinaus gibt es aber in der Praxis auch (vom Standpunkt der Grundlagenforschung und einer Vergrößerung des Fundus an Erkenntnissen aus Projekten ohne Gewinnorientierung dringend erwünschte) klinische Studien, die ebenfalls der Definition einer klinischen Prüfung entsprechen, bei denen allerdings die Initiative von der ärztlichen Seite ausgeht. In einem solchen Fall sehen AMG und ICH-GCP-Guideline vor, daß der verantwortliche Arzt die Funktionen von Prüfer und Sponsor in Personalunion (Sponsor-Prüfer) vereint.

Wie bereits einleitend erwähnt, ist die Involvierung eines entsprechend qualifizierten Statistikers/Biometrikers in die Planung und Auswertung einer klinischen Prüfung sowohl im AMG als auch in den ICH-GCP-Guidelines festgeschrieben. Entsprechend dem zugrundeliegenden Konzept des mehrheitlichen Vorliegens eines potenten und fachkundigen Auftraggebers scheint die Tätigkeit des Biometrikers in den Kapiteln der Sponsorverantwortlichkeiten auf.

Für sowohl von ärztlicher Seite als auch durchaus von industrieller Seite initiierte klinische Prüfungen hat sich ein Kooperationsmodell bewährt, in dem der Biometriker neben Sponsor und Prüfer eine dritte gleichwertige Parteienrolle übernimmt. Die Vorteile eines derartigen 3-Parteien-Modells liegen vor allem in der ,neutraleren“ Positionierung des Biometrikers gegenüber dem Prüfer. Dadurch wird die ansonsten fast monopolartig dominierende Rolle des Sponsors bei Datenmanagement, Datenauswertung und Ergebnisdarstellung vermieden und eine objektivitätsfördernde Ausgewogenheit zwischen den beteiligten Parteien begünstigt.

Eine derartige partnerschaftliche Struktur, die sich auch bereits vielfach bewährt hat, erfordert sowohl eine klare Abgrenzung der Kompetenzen und Tätigkeiten aller Parteien zueinander (Definition von Schnittstellen) als auch die Gewährleistung einer gleichermaBen hochqualitativen Leistungserbringung durch alle Beteiligten. Diesen Anforderungen kann nur durch die Implementierung und Anwendung von SOPs durch alle drei Parteien entsprochen werden, auch wenn entsprechende gesetzliche Vorschriften nur für den Sponsor existieren.

Die juristisch gesehen auf freiwilliger Basis erfolgende, vom praktischen Standpunkt aus aber dringend empfehlenswerte Etablierung von SOPs bei Biometriker und Prüfer (u.a. Schimetta et al., 1996, Kolman et al., 1998) gewährleistet also den Status einer gegenüber dem Sponsor gleichwertig organisierten Partei, bringt Vorteile bei regelmäßigen Projektabwicklungen, bürgt für die Einhaltung eines einmal definierten Qualitätsstandards und bietet die Möglichkeit einer sukzessiven Verbesserung desselben.

\section{Kernbereiche von Sponsor-SOPs}

Sponsor-SOPs befassen sich ausführlich mit Planungs- und Qualitätssicherungsabläufen, aber auch mit gesetzlichen Verpflichtungen z.B. betreffend Versicherung, Meldewesen etc. Im Rahmen der Planungs-SOPs werden u.a. die Erstellung diverser Unterlagen (BAH 1998), die Auswahl von Prüfern und Prüfzentren, die Bereitstellung von Prüfmustern, aber auch die Auswahl von und der Umgang mit Auftragsforschungsinstituten (Contract Research Organisations, CROs), auf welche Verantwortlichkeiten des Sponsors teilweise oder gänzlich übertragen werden können, standardisiert. SOPs im Qualitätssicherungs- 
sektor betreffen u.a. diverse Kommunikationsabläufe, Dokumentations- und Archivierungspflichten, Monitoring und Audit sowie interne Verfahren zur Qualitätskontrolle (,InProzeß-Kontrollen“). Schließlich werden die Anforderungen an Biometrie und Datenmanagement entweder in Form von detaillierten Einzel-SOPs in das Gesamtpaket integriert oder - was im vorliegenden Artikel näher dargestellt werden sollte - in Form einer „Schnittstellen-SOP“ als generelles Anforderungsprofil für einen im Rahmen des diskutierten 3-Parteien-Modells partnerschaftlich eingebundenen Biometriker vorgegeben.

\section{Schnittstellen zur Biometrie}

Die erwähnte, vom Sponsor zu formulierende „Schnittstellen-SOP“ enthält sowohl die qualitativen Anforderungen an den Biometriker und dessen Umfeld (siehe Übersicht 1) als auch die von diesem gewünschten Aktivitäten (siehe Übersicht 2).

Übersicht 1: Anforderung an den Biometriker und dessen Umfeld:

- Ausbildung

- Erfahrung

- SOPs

- Ausrüstung

- Datenzugänglichkeit

- Vertraulichkeit

- Stellvertretung

- Verantwortlichkeit

- Auditierbarkeit

Übersicht 2: Gewünschte Aktivitäten des Biometrikers betreffend:

- Studienplanung

Fallzahlschätzung

statistische Methodik

Randomisierung

Stratifizierung

- Studienabwicklung

statistische Amendments

Zwischenauswertung

- Datenmanagement

Doppeleingabe

Vollständigkeit

Plausibilität

Auditierbarkeit

- statistische Analyse

festgelegte Auswertungen

bedarfsorientierte Zusatzanalyse 


\section{- Ergebnisdarstellung}

biometrische Inhalte des Abschlußberichts

biometrische Abschnitte der Publikation

Naturgemäß sind eine umfassende biometrische/statistische Ausbildung (z.B. Hochschulstudium bzw. Hochschulabschluß) und ausreichende Erfahrungen im Umgang mit klinischen Prüfungen (biometrische Planung und Auswertung mehrerer Studien) zu fordern. Aus bereits diskutierten Gründen hat die Qualitätssicherung und -standardisierung unter Zuhilfenahme von SOPs zu erfolgen. Ausreichende Ressourcen bzw. Ausstattungen bezüglich biometriespezifischer Hardware und Software verstehen sich von selbst.

Die Spezifizierung der Datenzugänglichkeit (Beschränkung auf Berechtigte, Nachvollziehbarkeit von Zugriffen auf Daten) soll unzulässige Manipulationen verhindern, aber auch die Vertraulichkeit sämtlicher im Rahmen einer klinischen Prüfung bereitgestellten und anfallenden Informationen sicherstellen. Wesentlich ist auch die Etablierung eines Stellvertretungssystems für den Fall, daß der verantwortliche Biometriker oder andere Schlüsselpersonen in dessen Bereich kurzfristig oder längerfristig ausfallen (Vermeidung einer Projektgefährdung durch Personalausfälle). Ungeachtet des Umfangs seines Mitarbeiterstabs hat der Biometriker gegenüber dem Sponsor und dem Prüfer die volle Verantwortung für die gesamten Teamtätigkeiten zu übernehmen (dieselbe hierarchische Struktur gilt auch für Sponsor und Prüfer). Schließlich sollte das Einverständnis bestehen, so wie im Sponsoren- und im Prüferbereich auch im biometrischen Bereich allfällige Audits und Inspektionen zu akzeptieren.

Im Rahmen eines Studienablaufs werden die ersten Tätigkeiten des Biometrikers bereits in der Planungsphase benötigt. Hier geht es insbesondere um die Erstellung der Prüfplanabschnitte mit statistischer Ausrichtung wie Fallzahlschätzung, statistische Methodik, Signifikanz und Power, Randomisierung und Stratifizierung, aber auch um die Begutachtung von Prüfplanabschnitten mit Verzerrungspotentialen und möglichen Auswirkungen auf biometrische Details (z.B. Überprüfung von Design, Zielparametern, Versuchspersonenselektion, Drop-Out-Definitionen, Abbruchkriterien etc.).

Während der Studienabwicklung bzw. Medikationsphase sind allfällige Amendments je nach Ausmaß der statistischen Inhalte und Konsequenzen zu verfassen oder zu begutachten sowie allfällige (geplante oder aufgrund medizinischer Einschätzungen aktuell geforderte) Zwischenauswertungen vorzunehmen.

Eine oftmals nicht im biometrischen Kompetenzbereich angesiedelte Tätigkeit ist das Datenmanagement (die Aufbereitung der dokumentierten Einzeldaten für die statistische Auswertung). Für eine entsprechende Verantwortungsübernahme des Biometrikers spricht, daß bereits früh eine Vertrautheit mit dem Datenmaterial entsteht, eventuelle SoftwareInkompatibilitäten (Masken, Programme) vermieden werden und schließlich verschiedene Plausibilitätschecks (z.B. Ausschluß von Betrug) schon in einem initialen Bearbeitungsstadium kompetent vorgenommen werden können. $\mathrm{Zu}$ fordern ist im Rahmen der Abwicklung des Datenmanagements eine Doppeleingabe der erhobenen Einzeldaten durch zwei verschiedene Personen, eine Überprüfung des Datenmaterials auf Vollständigkeit und Plausibilität (mit Inkludierung entsprechender Maßnahmen zur Erhöhung der Datenqualität) und die Durchführung sämtlicher Datenbearbeitungen in nachvollziehbarer Form (Konzeption eines Audit-Trails). 
Die statistische Analyse bezieht sowohl die prospektiv im Prüfplan festgelegten Auswertungen als auch bedarfsorientierte Zusatzanalysen (auch wenn die Wertigkeit von letzteren oftmals niedrig ist) mit ein.

Bei der Ergebnisdarstellung hat der Biometriker die Verantwortung für die Formulierung sämtlicher einschlägigen Inhalte des Abschlußberichts (u.a. geplante und gewählte Auswertungsverfahren, Behandlung von Missing Data und Drop Outs, Auswertungs- und Zwischenauswertungsergebnisse, Begründung allfälliger Abweichungen von prospektiven Verfahrensfestlegungen, Ergebnisinterpretation aus biometrischer Sicht, Einzeldatenlistings etc.). Auch die Mitwirkung an einer Ergebnispublikation in Form einer Abfassung von deren statistischen Abschnitten und einer generellen Begutachtung der Inhalte gehört zu den gewünschten Leistungen.

\section{Fazit}

Die in den letzten Jahren stark gestiegenen Anforderungen an die Qualität klinischer Prüfungen führten zu einem erhöhten Stellenwert der Biometrie. Die Formalpflichten einer Coautorschaft bei Prüfplan, Abschlußbericht und Publikation sind Ausdruck einer gewünschten hohen Mitverantwortlichkeit des Biometrikers, die klar über eine Einbringung der Biometrie als Hilfswissenschaft bzw. als begrenzte Serviceleistung hinausgeht.

Demgemäß stellt es für den Biometriker eine Notwendigkeit dar, dieser neuen Situation Rechnung zu tragen, indem er das seitens des Sponsors in einer Schnittstellen- SOP präzise formulierte Wunschprofil bezüglich seiner Voraussetzungen und Tätigkeiten in die Praxis umsetzt.

Als Instrument für diese Praxisumsetzung sind spezifische Biometrie-SOPs auf Grundlage der ICH Guideline for Statistical Principles for Clinical Trials (E9) wesentlich, wenn nicht unerläßlich. Als Beispiel sei Schmoor et al. (1997) angeführt. Bringt sich der Biometriker somit als gleichwertiger Partner in die institutionalisierte Konstellation von Prüfer und Sponsor mit ein, so entsteht ein qualifiziertes, effizientes und somit für alle Beteiligten attraktives System für die interdisziplinäre Abwicklung klinischer Prüfungen.

\section{Literatur}

Arzneimittelgesetz BGBl. 1983/185 idF BGBl. 1994/107.

Bundesfachverband der Arzneimittel-Hersteller e.V. (BAH). Standardverfahrensanweisung (SOP) der fiktiven Firma „Muster“. Erstellung von Prüfplänen für klinische Prüfungen. Krahe Druck, Unkel, 1998.

EC-GCP-Note for Guidance: Good Clinical Practice for Trials on Medicinal Products in the European Community. In Klinische Arzneimittelprüfungen in der EG, Ed.-CantorVerlag, Aulendorf, 1992.

ICH Harmonised Tripartite Consolidated Guideline for Good Clinical Practice (E6), Mai 1996. 
ICH Harmonised Tripartite Guideline for Statistical Principles for Clinical Trials (E9), Jänner 1997.

J. Kolman, P. Meng and G. Scott. Good Clinical Practice. Standard Operating Procedures for Clinical Researchers. John Wiley \& Sons, Chichester, 1998.

W. Schimetta, G. Pölz, W. Pölz und F. Aichner. Standard Operating Procedures (SOPs) für an klinischen Prüfungen nach dem österreichischen Arzneimittelgesetz beteiligte Prüfer. GFI Gesellschaft für medizinische Information mbH, 1996.

C. Schmoor, C. Eisele, E. Graf, W. Sauerbrei, B. Klingele, A. Hellmer, R. Rossner und M. Schumacher. Arbeitsweisen des Methodischen Zentrums am Institut für Medizinische Biometrie und Medizinische Informatik der Universität Freiburg bei der biometrischen Betreuung klinischer Studien. Informatik, Biometrie und Epidemiologie in Medizin und Biologie, 28(4):253-274, 1997.

Adresse der Autoren:

Werner Pölz, Wolfgang Schimetta, Gabriele Pölz

Institut für Systemwissenschaften

Universität Linz

Altenbergerstraße 69

A-4040 Linz, Austria

Tel.: +43732 2468/851

Fax: +43732 2468/798

E-mail: Werner.Poelz@jk.uni-linz.ac.at

Web: http://www.sea.uni-linz.ac.at/statistik/homeAsoklif.htm 